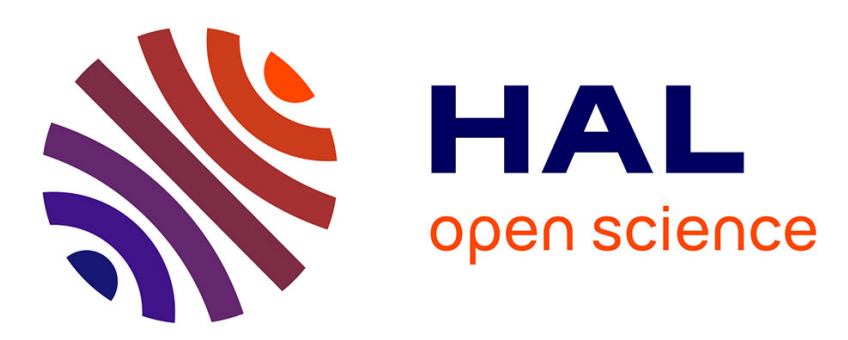

\title{
A multi-scale model for fluid transport through a bio-inspired passive valve
}

Simon Gravelle, Jacques Dumais

\section{To cite this version:}

Simon Gravelle, Jacques Dumais. A multi-scale model for fluid transport through a bio-inspired passive valve. Journal of Chemical Physics, In press, 10.1063/1.5126481 . hal-02424741

\section{HAL Id: hal-02424741 \\ https://hal.science/hal-02424741}

Submitted on 28 Dec 2019

HAL is a multi-disciplinary open access archive for the deposit and dissemination of scientific research documents, whether they are published or not. The documents may come from teaching and research institutions in France or abroad, or from public or private research centers.
L'archive ouverte pluridisciplinaire HAL, est destinée au dépôt et à la diffusion de documents scientifiques de niveau recherche, publiés ou non, émanant des établissements d'enseignement et de recherche français ou étrangers, des laboratoires publics ou privés. 


\title{
A multi-scale model for fluid transport through a bio-inspired passive valve
}

\author{
Simon Gravelle*1,2 and Jacques Dumais ${ }^{1}$ \\ ${ }^{1}$ Facultad de Ingeniería y Ciencias, Universidad Adolfo Ibáñez, Viña del Mar, Chile \\ ${ }^{2}$ School of Engineering and Material Science, Queen Mary University of London, London, United Kingdon $*$
}

Tillandsia landbeckii is a rootless plant thriving in the hyper-arid Atacama Desert of Chile. These plants use unique cellulose-based microscopic structures called trichomes to collect fresh water from coastal fog. The trichomes rely on a passive mechanism to maintain an asymmetrical transport of water: they allow for the fast absorption of liquid water deposited by sporadic fog events while preventing evaporation during extended drought periods. Inspired by the trichome's design, we study fluid transport through a micrometric valve. Combining Grand Canonical Monte Carlo with Non-Equilibrium Molecular Dynamics simulations, we first analyze the adsorption and transport of a fluid through a single nanopore at different chemical potentials. We then scale up the atomic results using a lattice approach, and simulate the transport at the micrometric scale. Results obtained for a model Lennard-Jones fluid and TIP4P/2005 water were compared, allowing us to identify the key physical parameters for achieving a passive hydraulic valve. Our results show that the difference in transport properties of water vapor and liquid water within the cellulose layer is the basis for the ability of the Tillandsia trichome to function as a water valve. Finally, we predict a critical pore dimension above which the cellulose layer can form an efficient valve.

The following article has been accepted by the Journal of Chemical Physics. After it is published, it will be found at $10.1063 / 1.5126481$

\section{INTRODUCTION}

A large fraction of water on Earth is, from a thermodynamic point of view, degraded as compared to pure liquid water [1. Most water is either mixed with salt or in the vapor phase. Numerous methods were developed to extract fresh water from sea water, including thermal methods such as distillation and pervaporation [2] and non-thermal methods such as reverse osmosis [3, 4]. While sea water and its purification are already the focus of intensive research activity, another exciting but neglected research direction concerns the atmospheric water vapor. Gaining access to this source of water is appealing as it is available anywhere on the Earth's surface, even in arid regions such as the Atacama Desert of Chile. In those regions, rainfalls are rare thus making atmospheric water the most important source of water. Moreover, at many sites, the relative humidity reaches saturation on a regular basis as a consequence of nightly radiative cooling [5], allowing liquid water to appear cyclically in the form of fog or dew [6, 7]. Yet, collecting water from the atmosphere is a challenge as this source of water is diffuse, and extensive structures are needed to collect acceptable amounts [8]. Following capture, an equally important challenge is to prevent the stored water from evaporating during prolonged dry periods.

Collecting water while preventing its evaporation is a challenge that desert plants face daily. One species in particular, Tillandsia landbeckii, thrives in the Atacama Desert of Chile, despite facing extreme climate conditions
10]. T. landbeckii plants are rootless and rely almost exclusively on the fog captured by their leaves to satisfy their water needs [11, 12. Fog events deposit fine water droplets on a layer of hydrophilic hairs called trichomes. The liquid water is then conducted toward the plant cells through the trichome stalk (Fig[1]A). Water absorption is driven by osmosis, thanks to a favorable chemical potential gradient between the deposited fog water and the plant cells [13, 14]

$$
\Delta \mu_{\text {abs }}=\mu_{\text {fog }}-\mu_{\text {cell }} \approx 22 \mathrm{~J} / \mathrm{mol},
$$

where $\mu_{\text {cell }} \approx-22 \mathrm{~J} / \mathrm{mol}$ is the chemical potential inside the plant cells [9] and $\mu_{\text {fog }} \approx 0$ is the chemical potential of the almost pure water of the fog droplets. Note that we use the potential of pure water at ambient temperature and pressure as the reference chemical potential. A typical liquid water absorption flow rate as measured for the Tillandsia epidermis is $Q_{\text {abs }} \approx 2.2 \mathrm{~mol} / \mathrm{cm}^{2} / \mathrm{s}[9$. Based on the flow rate and the chemical potential drop, the resistance of the plant epidermis to the absorption of liquid water is:

$$
\mathcal{R}_{\mathrm{abs}}=\frac{\Delta \mu_{\mathrm{abs}}}{Q_{\mathrm{abs}}} \approx 10 \mathrm{~J} \mathrm{~s} \mathrm{~cm}^{2} / \mathrm{mol}^{2} .
$$

Between fog events, the plant is exposed to dry air, and evaporation occurs through the trichomes of the epidermis. Assuming a relative humidity of $\mathrm{RH}=0.5$, the chemical potential drop between the plant cells and the dry air is

$$
\Delta \mu_{\text {eva }}=\mu_{\text {air }}-\mu_{\text {cell }} \approx-1708 \mathrm{~J} / \mathrm{mol},
$$

where the air chemical potential is $\mu_{\text {air }}=R_{g} T \ln (\mathrm{RH}) \approx$ $-1730 \mathrm{~J} / \mathrm{mol}$, with $T=300^{\circ} \mathrm{K}$ the temperature, and $R_{g}$ the gas constant [15]. A typical water evaporation flow

* simon.gravelle@live.fr 
(a)

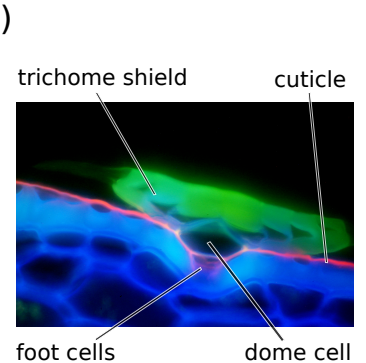

(b)
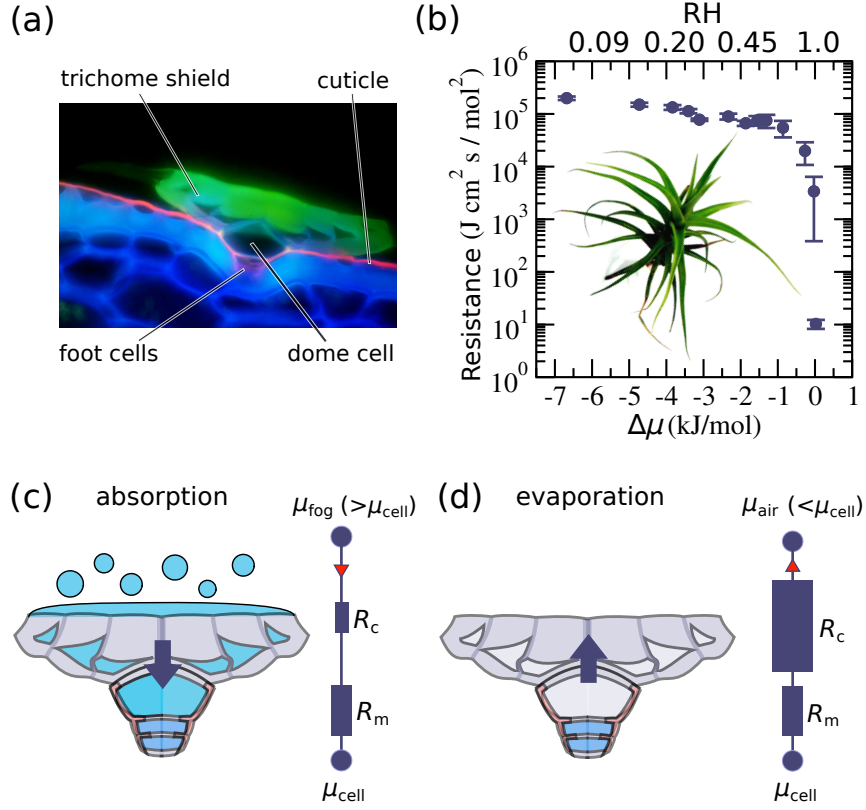

FIG. 1. (A) Micrograph of the Tillandsia trichome, with the trichome shield in green, the plant cells in blue, and the quasi-impermeable waxy cuticle in red. (B) Resistance to water transport of Tillandsia plant as a function of the difference in chemical potential between the atmosphere and the plant cells, see text for details. The point on the right is liquid absorption $(\Delta \mu>0)$, the others are evaporation $(\Delta \mu<0)$, data extracted from Ref. [9]. (C) Scheme of the trichome during liquid water absorption, with liquid water filling the cellulose of the shield and the dome cell. Insert: electrical scheme associated with Tillandsia trichome during absorption, with $R_{c}$ and $R_{m}$ the resistance to water transport of the cellulose shield and the cell membranes respectively, $\mu_{\text {fog }}$ the chemical potential of the fog, and $\mu_{\text {cell }}$ the chemical potential of the cells. (D) Trichome during water evaporation with associated electrical scheme and $\mu_{\text {air }}$ the chemical potential of the air.

rate as measured for the Tillandsia epidermis is $Q_{\text {eva }} \approx$ $-0.02 \mathrm{~mol} / \mathrm{cm}^{2} / \mathrm{s}$ [9]; that is, two orders of magnitude lower (in absolute value) than the liquid water absorption flow rate. We define the water flux asymmetry coefficient as

$$
\alpha_{Q}=\left|\frac{Q_{\text {abs }}}{Q_{\text {eva }}}\right| \approx 110
$$

and the resistance that the plant epidermis offers to water evaporation (for $\mathrm{RH}=0.5$ ) is

$$
\mathcal{R}_{\text {eva }}=\frac{\Delta \mu_{\text {eva }}}{Q_{\text {eva }}} \approx 8.5 \cdot 10^{4} \mathrm{~J} \mathrm{~s} \mathrm{~cm}^{2} / \mathrm{mol}^{2},
$$

so the plant offers a resistance to evaporation that is almost four orders of magnitude larger than the resistance to liquid water absorption (Eq. (2)). Moreover, the resistance that the Tillandsia epidermis offers to water exchanges with the environment varies with the relative humidity of the atmosphere. The resistance increases non-linearly with decreasing external chemical potential and reaches its highest value for the lowest relative humidity (Fig11B).

Remarkably, Tillandsia's ability to quickly absorb liquid water while blocking its evaporation is not based on the actuation of a valve, but relies on a passive mechanism originating in the trichome structure [9]. In short, when entering (or escaping) the plant through the trichome, water crosses three major elements; the trichome shield, the lumen of the dome cell, and the semi-permeable membranes of the cells at the trichome foot (Fig11A, C, D). Neglecting the resistance offered by the dome cell, as well as the resistance offered by the boundary layer [9, 15, the hydrodynamic resistance of the trichome is the sum of two contributions: (i) the resistance of the foot cell's membranes, (ii) and the resistance of the trichome shield, which is made of cellulose. As will be discussed below, the resistivity of cellulose-based materials to water transport varies by orders of magnitude with the water content [16]. Therefore, the trichome shield offers a large resistance to water vapor when the environment is dry and a low resistance to liquid water when the environment is wet [9]. Assuming that the resistance of the foot cell's membrane is constant irrespective of the external relative humidity, the main resistance a trichome opposes to water evaporation comes from the shield, and the main resistance to liquid water absorption comes from the foot cell's membrane (Fig[1]C,D). Qualitatively similar results have been obtained with a biomimetic system made of a commercial semi-permeable membrane in series with a layer of cellulose [9].

The variation of the trichome resistance with the water content of the shield is a key ingredient allowing the trichome to function as a valve. This response has several potential explanations: (i) the competition between the different transport regimes, respectively liquid phase transport, capillary transport, and vapor diffusion [17, (ii) the presence of a bound phase of slowly diffusing water molecules alternating between diffusion and adsorption in a "stop-and-go" motion [18, 19, and (iii) the hygroscopic structure of the cellulose that swells with its water content, thus offering smaller and less interconnected pores for the transport of vapor as compared with the transport of liquid water [20].

In this work, we use numerical calculations to model fluid transport through an ideal porous medium in contact with a reservoir with a broad range of chemical potential values, mimicking the situation faced by the Tillandsia trichomes. In order to accurately predict fluid transport at the micrometric scale, while also capturing the subtle effects of confined fluid such as capillary condensation and Knudsen diffusion, we first perform atomic simulations of fluid adsorption and transport through a single nanopore, 
and then scale up the atomic results using a bottomup model. As an initial step, benchmark simulations using a model Lennard-Jones (LJ) fluid are performed. Results obtained under this hypothesis show that a porous medium cannot work as a hydraulic valve for a LJ fluid. We then perform similar simulations but replace the LJ fluid with water. With this new hypothesis, we find that a porous medium can work as a hydraulic valve similar to the Tillandsia trichomes. Comparison between the two fluids allows us to identify the key fluid properties at the origin of the valve function. For the sake of simplicity, the porous medium is made of an assembly of cylinders of given radius. The simple model is not aimed at predicting quantitatively the permeability of a complex multi-scale matrix such as cellulose but will allow us to compare flow rates obtained for different fluid contents and different pore sizes. We will thus be able to identify the essential physical parameters for the implementation of a passive valve.

\section{MODEL FLUID (LENNARD-JONES)}

\section{A. Transport through a single pore}

Using LAMMPS 21, we perform atomic simulations of the adsorption and transport of a Lennard-Jones fluid in a model pore with adsorbing wall. To do so, we combine Grand Canonical Monte Carlo simulations (GCMC) with Non-Equilibrium Molecular Dynamics (NEMD) 22. Particles interact solely through a LJ potential $U(r)=4 \epsilon\left[(\sigma / r)^{12}-(\sigma / r)^{6}\right]$ for $r<r_{c}$, where $\epsilon$ is the depth of the potential well, $\sigma$ is its cross-section, and $r_{c}=2.5 \sigma$ is the interaction cut-off. The pore is a cylinder of radius $R$ and length $L$, that is made effectively infinite by the use of periodic boundary conditions. We made sure that the choice of the length $L$ has no impact on the results of the simulation by choosing $L$ much larger than twice the interaction cut-off, hence avoiding interactions between mirror images through the periodic boundary conditions. In order to prevent the adsorbed phase from modifying the effective hydrodynamics radius of the pore, and also avoid the migration of the adsorbed particles [23, the pore is built as follows. The cylinder's wall is made of three layers: two internal "solid" layers with fluid-solid interactions parameters $\sigma_{\mathrm{fs}}=\sigma$ and $\epsilon_{\mathrm{fs}}=0.2 \epsilon$, and one external "adsorption" layer, with $\sigma_{\text {ads }}=\sigma$ and potential well $\epsilon_{\text {ads }}$. Simulations with two different values for $\epsilon_{\mathrm{ads}}$ are performed, $\epsilon_{\mathrm{ads}}=0.2 \epsilon$ or $\epsilon_{\mathrm{ads}}=20 \epsilon$ respectively, allowing us to control the adsorption at the pore surface. LJ cross-parameters are computed using the Lorentz Berthelot mixing rules, and fluid-fluid interaction parameters $(\epsilon$ and $\sigma$ ) are used as reference energy and distance, respectively. Half the atoms of the internal layers are randomly deleted to provide roughness to the canal and ensure that the effective hydrodynamic radius of the cylinder remains constant regardless of the presence of adsorbed particles at the surface (Fig. 22A).
The external layer ensure the impermeability of the canal. The timestep is equal to $0.005 \tau$, where $\tau=\sigma \sqrt{m / \epsilon}$, where $m$ is the fluid particle mass.

First, the system is put into contact with a fictive reservoir of desired chemical potential $\mu^{*}=\mu / \epsilon$. The fluid particles are maintained at a temperature $T^{*}=k_{B} T / \epsilon=1$ by a canonical Nose-Hoover thermostat. We use GCMC algorithm, which consists in successive trial insertions and deletions of particles 24, 25. Once the concentration has reached equilibrium, the fluid density number $\rho^{*}=\rho \sigma^{3}$ is measured. We extract adsorption isotherms for different tube radius $R=10$, 20, and $40 \sigma$ (Fig. 2 B). Results show that the adsorption does not depend on the tube radius $R$. Note that the adsorption could depend on the pore's radius in the case of a LJ fluid, but in a different range of tube radius and/or different range of temperature [26. Results also show that isotherms strongly depend on the adsorption energy $\epsilon_{\text {ads }}$, because a large value of $\epsilon_{\text {ads }}$ leads to the formation of a layer of adsorbed fluid particles at the wall (Fig. 2 A, B).

The final states of GCMC simulations are used as initial states for NEMD simulations. Now with a fixed number of particle, a force $f_{0}=0.005 \epsilon / \sigma$ is applied on each fluid particle in the direction of the tube. The fluide particles are maintained at a temperature $T^{*}=1$ by a canonical Nose-Hoover thermostat applied to the degree of freedom normal to the direction of the force only. After an equilibration phase of $50000 \tau$, we record the average particle velocity in the direction of the force from which we evaluate the flux of particles $J^{*}=J \times \tau \sigma^{2}$. We then evaluate the pore permeability $K=J / f_{0}$, and reduced permeability $K^{*}=K \tau \sigma \epsilon$. Our results show that the permeability $K$ depends strongly on the tube radius $R$, as well as on the chemical potential $\mu$ (Fig.2 2 ). However, $K$ is independent on the number of adsorbed particles. Accordingly, we ignore the adsorbed particles in the following.

We now write an expression for the transport of the particles, taking into account both viscous and diffusive contributions [27]. In the limit of a large fluid density number $\rho$, the permeability of the nanopore is dominated by the viscous contribution

$$
K_{\eta}=\frac{R^{2} \rho^{2}}{8 \eta},
$$

where $\eta$ is the liquid viscosity, approximated as $\eta=$ $\eta_{0} \exp \left(\rho / \rho_{0}\right)$ with $\eta_{0}=0.013 \sqrt{m \epsilon} / \sigma^{2}$ and $\rho_{0}=0.17 \sigma^{-3}$, in qualitative agreement with the literature [28]. In the limit of a low fluid density number $\rho$, the permeability of the nanopore is dominated by the diffusive contribution

$$
K_{D}=\frac{D \rho}{R_{g} T},
$$

where $D$ is the particle diffusion coefficient. The diffusion coefficient $D$ is approximated as $D^{-1}=D_{\mathrm{K}}^{-1}+D_{0}^{-1}$, 
(a)

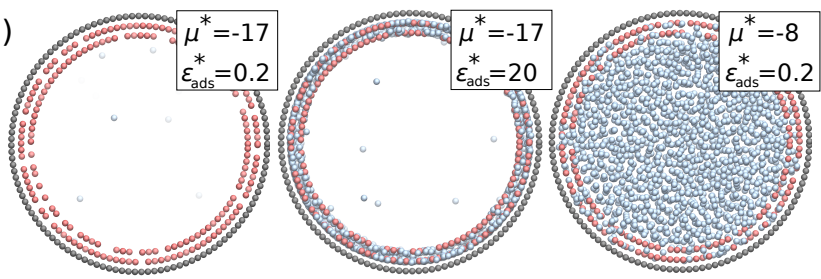

(b)
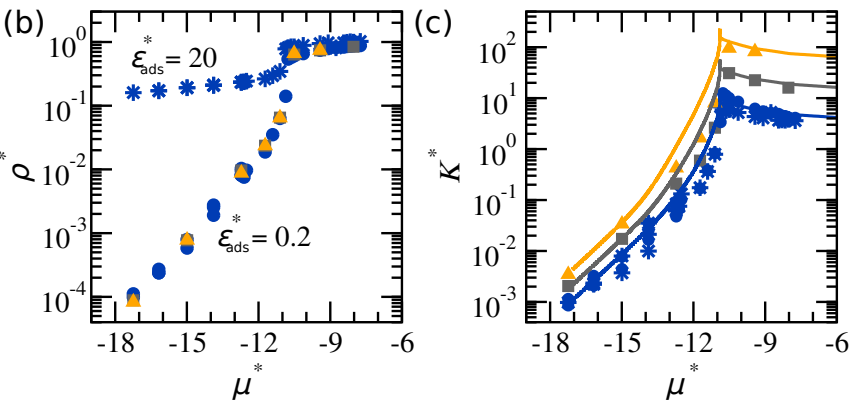

FIG. 2. Transport of a LJ fluid through a single nanopore. (A) Snapshot of the cross section of the cylinder of radius $R=10 \sigma$ for different values of the chemical potential $\mu^{*}$ and adsorption energy $\epsilon_{\text {ads }}^{*}$. The external adsorption layer is in gray, the solid atoms in red, and the fluid atoms in blue. (B) Adsorption isotherms for $\epsilon_{\mathrm{ads}}^{*}=0.2$ and three different tube radius, respectively $R=10 \sigma$ (blue disk), $R=20 \sigma$ (gray squares) and $R=40 \sigma$ (orange triangles), and for $\epsilon_{\mathrm{ads}}^{*}=20$ and $R=10 \sigma$ (blue stars). (C) Permeability corresponding to the panel $\mathrm{B}$, as a function of the chemical potential. Lines correspond to Eq. $(6)+$ Eq. (7).

where $D_{\mathrm{K}}$ is the concentration independent Knudsen contribution [29, 30] $D_{\mathrm{K}}=2 R u_{\mathrm{th}} / 3$, with $u_{\text {th }}$ the thermal velocity $u_{\mathrm{th}}=\sqrt{8 R_{g} T /\left(\pi M_{\mathrm{A}}\right)}$, and $D_{0}$ the concentration dependent contribution, $D_{0}=\lambda u_{\mathrm{th}} / 3$, where $\lambda^{-1}=\pi \sqrt{2} \rho \sigma^{2}$. Using the dusty gas formulation [31, we superpose the diffusive and viscous contributions from Eqs. (6) and (7) respectively, $K=K_{\eta}+K_{D}$, and find a good agreement with NEMD simulations (Fig. 2 C). The agreement is particularly good at high and low concentrations but deviate slightly from NEMD results at intermediate concentrations $\left(\rho^{*} \approx 0.1\right.$ and $\left.\mu^{*} \approx-12\right)$.

Now that we have extracted isotherms and transport properties for a single pore, we want to simulate fluid transport in the Tillandsia trichome at the micrometric scale. However, within a membrane with micrometric dimensions, large variations of fluid density can develop from one side to the other, particularly if the membrane separates a dry environment from a wet environment. In that case, the spatial concentration profile is non-trivial as a consequence of the nonlinearity between the fluid density, chemical potential, and pore permeability. In order to accurately predict fluid transport through a micrometric membrane, we scale up the molecular results obtained for a single nanopore. To do so, we use a bottom-up method in which a micrometric membrane is described as a bi-dimensional lattice. The adsorption and transport properties of each tile of the lattice are calculated from the GCMC/NEMD results (Fig.2), and the flux between neighboring tiles is calculated iteratively until equilibrium is reached.

\section{B. Transport through a micrometric medium}

In order to predict the permeability of a porous medium with micrometric dimensions, we scale up the GCMC-NEMD results into a lattice model 32, 33. The advantage of a bottom-up approach over the lattice Boltzmann method, for example 34, is that it requires no assumption on the phase of the fluid within the pore. The system is a lattice of total length $L_{\mathrm{m}}$, width $H_{\mathrm{m}}$, and depth $\ell$, divided into a $50 \times 50$ equal-size tiles. Each tile $i$ of size $\ell \times \ell \times \ell$, with $\ell=2000 \sigma$, contains a number $N_{i}$ of pores of radius $R_{i}$ and length $\ell$, with $R_{i}$ distributed as a Gaussian of mean $R$ and standard deviation $\Delta R$. The number of pore $N_{i}$ is chosen to ensure an effective porosity $\epsilon_{\mathrm{m}}=0.5$ through $\epsilon_{\mathrm{m}}=N_{i} \pi R_{i}^{2} / \ell^{2}$. The tortuosity is $\tau_{\mathrm{m}}=1$. Each tile $i$ is associated with a fluid density value $\rho_{i}$, a chemical potential value $\mu_{i}$, and a permeability value $M\left(N_{i}, R_{i}, \mu_{i}\right)$. The chemical potential is imposed on both left and right boundaries, respectively $\mu_{\text {left }}$ and $\mu_{\text {right }}$, using extra rows of sites at each end of the lattice and playing the role of bulk reservoirs (Fig.3A).

The respective chemical potentials of the right and left reservoirs were chosen in order to mimic the situation encountered in the Tillandsia trichome. The left row mimics the osmotic reservoir (the plant cells) and is maintained at a constant chemical potential $\mu_{\text {left }}=-5 \epsilon$. The right row mimics the environment whose chemical potential is varied from $\mu_{\text {right }}=-4.9 \epsilon$ (close to pure liquid) to $\mu_{\text {left }}=-20 \epsilon$ (vapor). Hence, the ratio between the chemical potential difference during evaporation and absorption respectively, $\Delta \mu_{\mathrm{eva}} / \Delta \mu_{\mathrm{abs}}=-150$, is equal to the one faced by Tillandsia plant when alternatively exposed to pure liquidwater and atmospheric relative humidity $\mathrm{RH}=0.25$. An initial linear gradient of chemical potential going from $\mu_{\text {left }}$ to $\mu_{\text {right }}$ is set through the system, and the initial density $\rho_{i}$ within each tile is calculated from the initial chemical potential using the adsorption isotherm obtained for $\epsilon_{\text {ads }}=0.2 \epsilon($ Fig. 2 2 B).

Following the approach of Botgan et al. [33, the time iteration consists in two steps. First the change in the local density $\rho_{i}$ for each tile $i$ is evaluated by considering incoming and outgoing fluxes from and to adjacent neighbors

$$
\frac{\partial \rho_{i}}{\partial t}=\sum_{a} M\left(N_{i}, R_{i}, \mu_{i}\right) \frac{\mu_{i+a}-\mu_{i}}{\ell}
$$

where $M\left(N_{i}, R_{i}, \mu_{i}\right)$ is the permeability of the tile $i . M$ is a function of the pore number $N_{i}$, the pore radius $R_{i}$ 

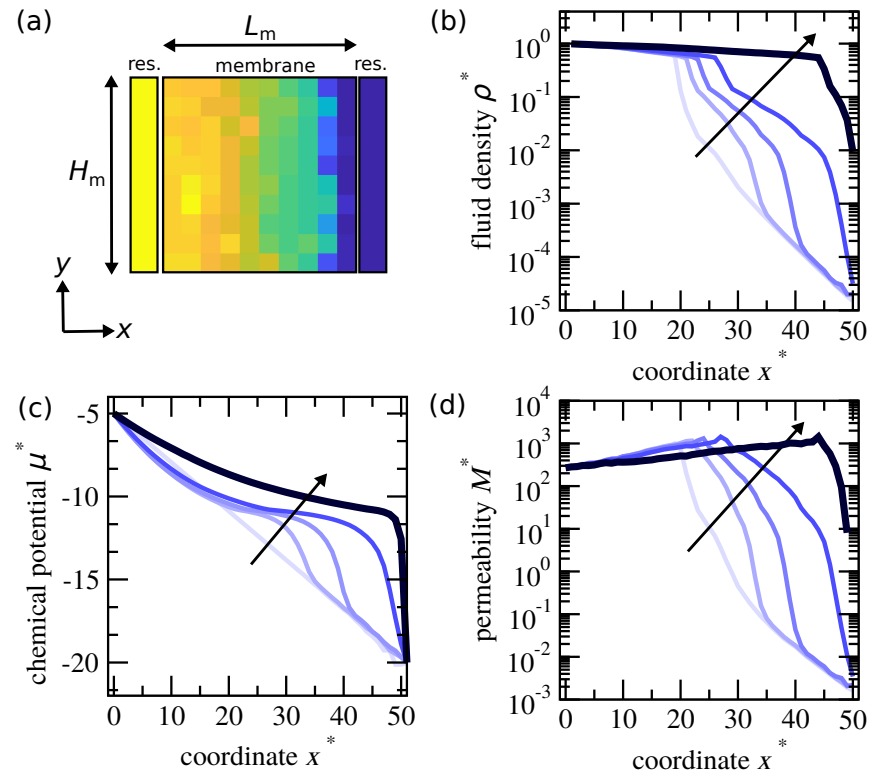

FIG. 3. (A) Scheme of the system with two reservoirs and membrane divided in tiles. The color field is the concentration, from high concentration in yellow to low concentration in blue. The number of tiles has been reduced for clarity. (B) y-averaged density $\rho^{*}=\rho \sigma^{3}$ as a function of the coordinate $x^{*}=x / \sigma$, from the initial state (light blue) to the final state (black line), for $L_{\text {lat }}=H_{\text {lat }}=10^{5} \sigma, \mu_{\text {left }}=-4.9 \epsilon$ and $\mu_{\text {right }}=-20 \epsilon$, and $R=100 \sigma$ and $\Delta R=10 \sigma$. The arrow shows the time evolution. (C) Corresponding y-averaged chemical potential $\mu^{*}=\mu / \epsilon$. (D) Corresponding y-averaged permeability $M^{*}=M \sigma \epsilon \tau$.

and the local chemical potential $\mu_{i}$, and is calculated from the permeability of a single pore as given by Eqs. (6) and (7). Then, the local chemical potential $\mu_{i}$ is updated with respect to the calculated concentration $\rho_{i}$, according to the adsorption isotherm obtained from GCMC simulations (for $\epsilon_{\mathrm{ads}}=0.2 \epsilon$ ) (Fig.2B). Eventually, the density, the chemical potential, and the permeability, all reach equilibrium (Fig.33). At equilibrium, the flux of liquid per unit of surface $Q^{*}=Q \sigma^{2} \tau$ in the $x$ direction is a constant along $x$ through the entire membrane (flux conservation).

We measure $Q$ as a function of the pore radius, in both liquid absorption $\left(\mu_{\text {right }}=-5 \epsilon\right)$ and evaporation $\left(\mu_{\text {right }}=-20 \epsilon\right)$ situations. Results show a monotonic increase of the flux with the average pore radius $R$ (Fig. 44A). The flux during absorption is about two orders of magnitude lower than during evaporation. The flux asymmetry coefficient $\alpha_{Q}=\left|Q_{\text {abs }} / Q_{\text {eval }}\right|$ (Eq. (4) remains quasi constant with the average pore radius $R$, and is about $\alpha_{Q} \approx 0.008$ (Fig. $4 \mathrm{~B}$ ).

We also measure the flux for different external potentials $\mu_{\text {right }}$. Results show a quasi linear dependence on $\Delta \mu=\mu_{\text {right }}-\mu_{\text {left }}$ for $\Delta \mu>-6 \epsilon$, and a plateau for lower values of $\Delta \mu$ (Fig. 4 C). Accordingly, the flux asymmetry
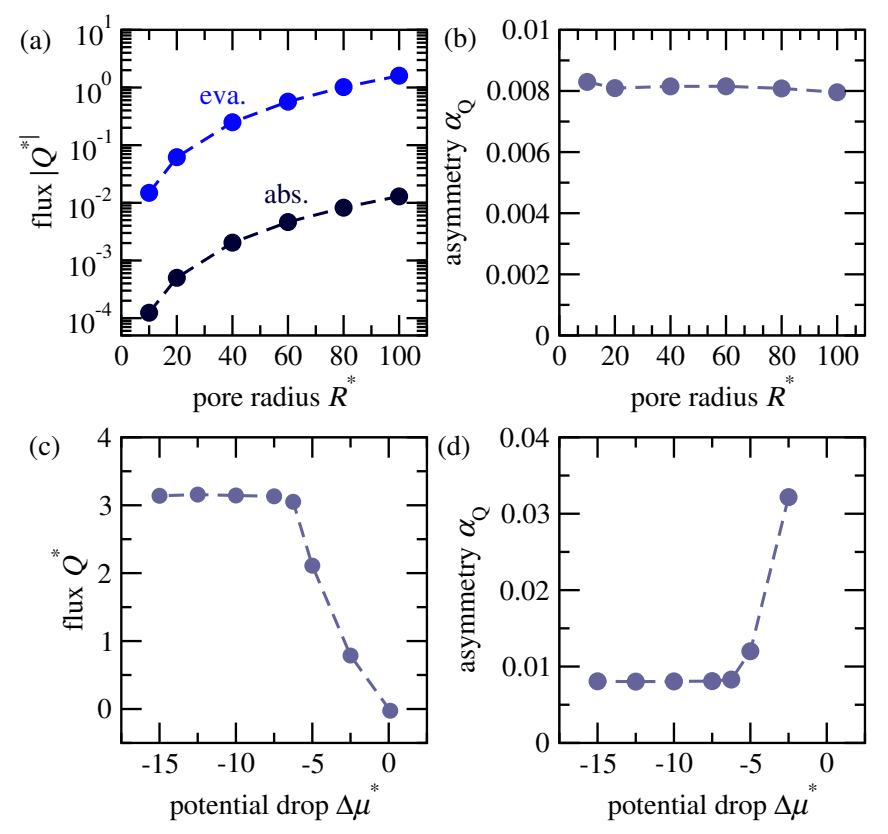

FIG. 4. Transport of LJ fluid through a micrometric medium (A) Absolute value of the flux $Q^{*}=Q \sigma^{2} \tau$ in absorption $\left(\mu_{\text {right }}=-5 \epsilon\right)$ and evaporation $\left(\mu_{\text {right }}=-20 \epsilon\right)$ as a function of the average pore radius $R^{*}=R / \sigma$. (B) Flux asymmetry coefficient $\alpha_{Q}=\left|Q_{\text {abs }} / Q_{\text {eva }}\right|$ with here $Q_{\text {eva }}=Q\left(\mu_{\text {right }}=-20 \epsilon\right)$ and $Q_{\text {abs }}=Q\left(\mu_{\text {right }}=-5 \epsilon\right)$ as a function of the pore radius. (C) Flux as function of the potential drop $\Delta \mu=\mu_{\text {right }}-\mu_{\text {left }}$ with $\mu_{\text {left }}=-4.9 \epsilon$ for a pore radius $R=100 \sigma$. (D) Flux asymmetry coefficient as a function of the potential drop. Here $Q_{\text {eva }}=Q\left(\mu_{\text {right }}\right)$

coefficient reaches a plateau for low chemical potentials (Fig.4 4 D). In addition, we also found that the flux scales inversely with the membrane length $Q \propto 1 / L_{\mathrm{m}}$ (not shown), as expected, with no impact on the asymmetry coefficient.

Altogether, our results show that a model porous medium forms an inefficient flow rectifier for a LJ fluid, with an asymmetry coefficient $\alpha_{Q}<1$. We have verified that a different choice of chemical potential for the osmotic reservoir $\left(\mu_{\text {left }}\right)$ leads to qualitatively similar results. An asymmetry coefficient inferior to one indicates that the system loses fluid through evaporation at a much higher rate than it absorbs it, in contrast with Tillandsia plants that show an asymmetry coefficient of $\alpha_{Q} \approx 110$ for water. But water differs from a LJ fluid [35, in particular because of electrostatic interactions and hydrogen bonding between water molecules. Hence, we now concentrate on the transport of water following the same procedure as the one followed for the LJ fluid. We also use a slightly more realistic cellulose model for the pore's wall but conserve the simple cylindrical geometry. 


\section{WATER}

\section{A. Transport through a single pore}

Now we study the transport of TIP4P/2005 water [36] using the code LAMMPS 21]. The wall of the cylindrical pore are made of amorphous cellulose, and we use the force field GLYCAM06 [37]. The initial coordinates of cellulose $\mathrm{I} \beta$ crystal structure are build using Cellulose-builder [38] and follow the crystallographic structure reported by Nishiyama et al. 39]. The $\mathrm{I} \beta$ fiber is replicated in all three directions of space, and a two-stages procedure of amorphization inspired by the work of Kulasinski et al. is performed [40, using a timestep of $0.1 \mathrm{fs}$. The polymer temperature is increased to reach $700 \mathrm{~K}$ in the isothermal-isobaric (NPT) ensemble during $1 \mathrm{~ns}$, at a pressure of $1 \mathrm{bar}$. Then the polymer is quenched to a temperature of $300 \mathrm{~K}$ and a pressure of 1 Bar and equilibrated for an additional 10 ns. Finally, the cellulose is frozen to prevent coupling between the cellulose structure and water. The validity of simulations performed using frozen walls is discussed below. The fibers contained within a cylinder of radius $R$ are removed to create the nanopore, with $R=0.5$, 0.8 , and $1.5 \mathrm{~nm}$, respectively. Each pore is replicated three times with different initial conditions in order to perform independent sets of simulations. The final total number of atoms of cellulose are typically 250, 1000, and 1700 , simulation box sizes are $1.5 \times 1.5 \times 1.5 \mathrm{~nm}^{3}$, $3 \times 3 \times 1.5 \mathrm{~nm}^{3}$, and $4 \times 4 \times 2 \mathrm{~nm}^{3}$, and the porosities are $\epsilon_{p}=0.35,0.22$, and 0.44 , respectively (Fig.5A). Water molecules are held rigid using the SHAKE algorithm [41. Lennard-Jones cross-parameters between water and cellulose atoms are computed using the Lorentz Berthelot mixing rules.

Water molecules are inserted into the simulation box using the Grand Canonical ensemble Monte Carlo (GCMC) technique (Fig.5A). Molecules are maintained at a constant temperature $T=300 \mathrm{~K}$ using integration on NoseHoover thermostat in the canonical (nvt) ensemble, with a timestep of $2.0 \mathrm{fs}$. The density per unit volume of pore $\rho$ is measured for a chemical potential $\mu$ varying from $-11 \mathrm{Kcal} \mathrm{mol}^{-1}(\mathrm{RH} \approx 1)$ to $-12.5 \mathrm{Kcal} \mathrm{mol}^{-1}$ $(\mathrm{RH} \approx 0.07)(\mathrm{Fig} .5 \mathrm{~B})$. Larger pores of radius $R=0.8$ and $1.5 \mathrm{~nm}$ are filled with water for large chemical potential, and filled with vapor otherwise. The jump in concentration is due to capillary condensation of the water within the pore 42. The jump occurs at a relative humidity $\mathrm{RH}^{*}$, given by the Kelvin equation

$$
\ln \left(\mathrm{RH}^{*}\right)=\frac{-2 \gamma V_{w}}{R_{g} T R_{\mathrm{eff}}},
$$

where $V_{w}$ is the molar volume of water, $\gamma=69 \mathrm{mN} / \mathrm{m}$ is the TIP4P/2005 water surface tension [43], and $R_{\text {eff }}$ is the effective radius of the pore. Reasonable estimates of $\mathrm{RH}^{*}$ are obtained using $R_{\text {eff }} \approx R-\sigma_{w}$ where $\sigma_{w} \approx 3 \AA$ is the water molecule diameter. Note that Eq. (9) offers only a rough approximation of $\mathrm{RH}^{*}$ when applied to nanopores, and more complete equations accounting for the attractive potential energy from the pore wall [44] or a modification of the surface tension as characterized by the Tolman length could be used instead [45. However, given the pores irregularities and the uncertainty on the values of effective radius $R_{\text {eff }}$, we limit ourselves to the simplest formula. We have also neglected the possibility of hysteresis, i.e. different values of $\mathrm{RH}^{*}$ between absorption and evaporation [42, 46. From Eq. (9), we get $\mathrm{RH}^{*} \approx 0.43$ for $R=1.5 \mathrm{~nm}$ and $\mathrm{RH}^{*} \approx 0.13$ for $R=0.8 \mathrm{~nm}$. The smallest pore $(R=0.5 \mathrm{~nm})$ is always filled with liquid for the range chemical potentials considered, in agreement with the predicted $\mathrm{RH}^{*}<0.01$.

The final states of the GCMC simulations are used as initial states for the NEMD simulations. A force $f_{0}=42 \mathrm{~J} \mathrm{~mol}^{-1} \AA^{-1}$ is applied to water molecules in the direction of the tube. Molecules are maintained at a constant temperature $T=300 \mathrm{~K}$ using a canonical Nose-Hoover thermostat (nvt) applied to the degree of freedom normal to the direction of the force. The average molecule velocity in the direction of the force is recorded, from which the flux per surface unit $J$ is measured (in mol $/ \mathrm{s} / \mathrm{m}^{2}$ ). From the flux $J$, we calculate the permeability $K=Q / f_{0}\left(\mathrm{~mol}^{2} / \mathrm{s} / \mathrm{m} / \mathrm{J}\right)$ of the pore as a function of the chemical potential $\mu$ (Fig.5C). When the pores are filled with liquid water, the results are well fitted by the viscous equation (Eq. (6)) using the viscosity of the TIP4P/2005 water model $\eta=0.855 \mathrm{mPas}$ 47, the bulk density of liquid water $\rho=5.5 \cdot 10^{4} \mathrm{~mol} / \mathrm{m}^{3}$, and effective hydrodynamic radii $R_{\text {hyd }}=0.42,0.97$ and $1.6 \mathrm{~nm}$. When the pores are filled with vapor, the results are well fitted by the diffusive equation (Eq. (7)) where we use as density the equilibrium density of gas in the reservoir, i.e. ignoring the adsorbed phase in agreement with results obtained with LJ fluid showing that the adsorbed phase does not impact the transport (Fig.5 C).

\section{Validity of using frozen walls}

The use of frozen cellulose walls allowed us to prevent coupling between cellulose and water, as well as to reduce the computational cost of the simulations. However, the use of a rigid model is known to be incorrect in principle, particularly if one considers phenomena related to correlation function, such as diffusion coefficients [48. To test the validity of the results obtained with frozen walls (Fig. 5), we performed additional simulations using a flexible model of cellulose. In order to describe the coupled adsorption and swelling of the porous material, we used a hybrid MD/GCMC molecular simulation that consists in alternating between GCMC steps and MD steps performed in the isothermal-isobaric (npt) ensemble (see Refs. 46, 49 for details). We performed simulations using a pore of radius $R=0.8 \mathrm{~nm}$ for two values of the 

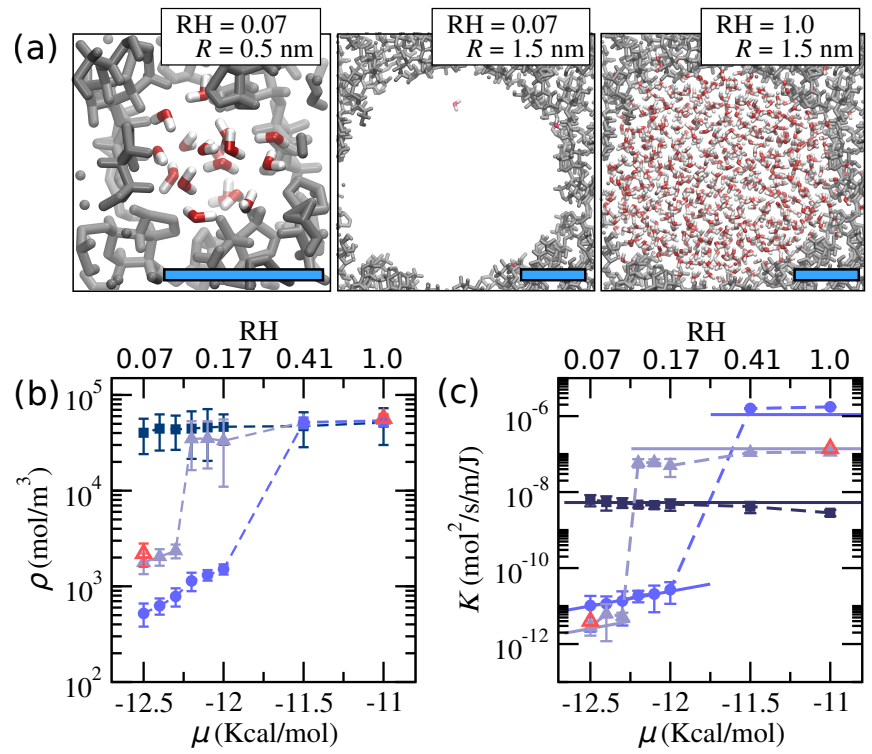

FIG. 5. Transport of water through a single nanopore. (A) Snapshot of the cross section of the nanopore of radius $R=$ $0.5 \mathrm{~nm}$ and a nanopore of radius $R=1.5 \mathrm{~nm}$ for different values of the chemical potential corresponding respectively to $\mathrm{RH}=$ 0.07 and 1.0. The cellulose is in grey, the oxygen and hydrogen atoms of the water molecules in red and white respectively. The blue scale corresponds to $1 \mathrm{~nm}$. (B) Adsorption isotherms of water in frozen cellulose pores for three different pore radii $R=0.5 \mathrm{~nm}$ (squares), $0.8 \mathrm{~nm}$ (triangles) and $1.5 \mathrm{~nm}$ (disks). Red open symbols are results obtained with flexible walls. (C) Water permeability $K$ of the cellulose pores as function of the chemical potential for the three radii.

chemical potential, $\mu=-12.5 \mathrm{Kcal} \mathrm{mol}^{-1}$ (pore filled with vapor), and $\mu=-11 \mathrm{Kcal} \mathrm{mol}^{-1}$ (pore filled with liquid). In the case of the pore filled with vapor, we observe a shrinking of the volume of the box by $\approx 8 \%$ with respect to the frozen case, and measure an equilibrium density of water $\rho=(2200 \pm 600) \mathrm{mol} / \mathrm{m}^{3}$, which corresponds to an increase of $\approx 25 \%$ with respect to the frozen walls case (red open symbol in Fig.5B). Then, the final state of the GCMC simulation is used as the initial state for a NEMD simulation during which the temperature of the flexible cellulose walls is maintained equal to $T=300 \mathrm{~K}$ using a canonical Nose-Hoover thermostat (nvt). From the NEMD simulation, we calculate the permeability of the pore and measure $K=(3.6 \pm 3.0) \cdot 10^{-12} \mathrm{~mol}^{2} / \mathrm{s} / \mathrm{m} / \mathrm{J}$ (red open symbols in Fig. 5 C). In the case of the pore filled with liquid $(\mu=-11 \mathrm{Kcal} / \mathrm{mol})$, we observe a swelling of the volume of the box by $\approx 2 \%$, and equilibrium density of $\rho=(2900 \pm 800) \mathrm{mol} / \mathrm{m}^{3}$, which corresponds to an increase of $\approx 6 \%$ with respect to the frozen walls case, and we then measure a permeability $K=(1.4 \pm 0.2) \cdot 10^{-7} \mathrm{~mol}^{2} / \mathrm{s} / \mathrm{m} / \mathrm{J}$ (red open symbol in Fig.5B, C). Alltogether, the results obtained with flexible walls show slight increases of both equilibrium density and permeability for both vapor-filled and liquid-filled pores, with respect to the results obtained with frozen walls. The similarities between the results suggest that the use of frozen walls is, in the present case,
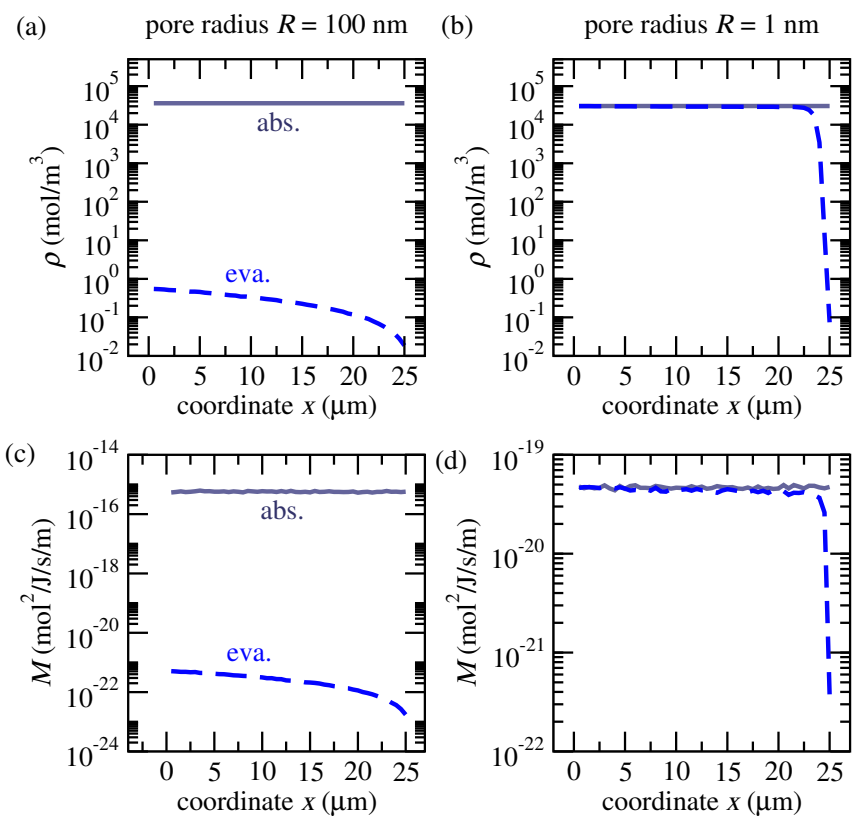

FIG. 6. Transport of water through a micrometric medium. (A) Density profile as a function of the $x$ coordinate for large average pore radius $(R=100 \mathrm{~nm})$, in evaporation $(\mathrm{RH}=0.01)$ (dashed line) and liquid adsorption $(\mathrm{RH}=1)$ (full line). (B) Density profile as a function of the $x$ coordinate for small average pore radius $(R=1 \mathrm{~nm})$. (C) Local permeability as function of $x$ for large average pore radius $(R=100 \mathrm{~nm})$ in both water evaporation and adsorption situations. (D) Local permeability as function of $x$ for large average pore radius $(R=100 \mathrm{~nm})$.

a good approximation for the study of transport through nanopores.

\section{B. Transport through a micrometric medium}

Following the same procedure as the one previously described, we scale up the GCMC-NEMD results obtained for water into a lattice model. The system is a lattice of total length $L_{\mathrm{m}}=25 \mu \mathrm{m}$, width $H_{\mathrm{m}}=L_{\mathrm{m}}$, and depth $\ell$, divided into a $50 \times 50$ equal-size tiles. Each tile is of size $\ell \times \ell \times \ell$ with $\ell=500 \mathrm{~nm}$. The chemical potential of the left row (osmotic reservoir) is equivalent to $\mathrm{RH}=0.991$, similar to the one measured on Tillandsia plant, and the external chemical potential is varied from dry $(\mathrm{RH}=0.01)$ to wet $(\mathrm{RH}=1)$.

We first analyze the water profile within a matrix of large pores of average radius $R=100 \mathrm{~nm}$ (pores radius are distributed as a Gaussian of standard deviation $\Delta R=10 \mathrm{~nm})$. When the system is put in contact with wet air $(\mathrm{RH}=1)$, the whole pore matrix is filled with liquid water of density $\rho \approx 5.5 \cdot 10^{4} \mathrm{~mol} / \mathrm{m}^{3}$ (full line, Fig. 6 A). When the system is put in contact with dry air $(\mathrm{RH}=0.01)$, the whole pore matrix is filled with water vapor of density $\rho<1 \mathrm{~mol} / \mathrm{m}^{3}$ (dashed line, Fig.6 A). As 

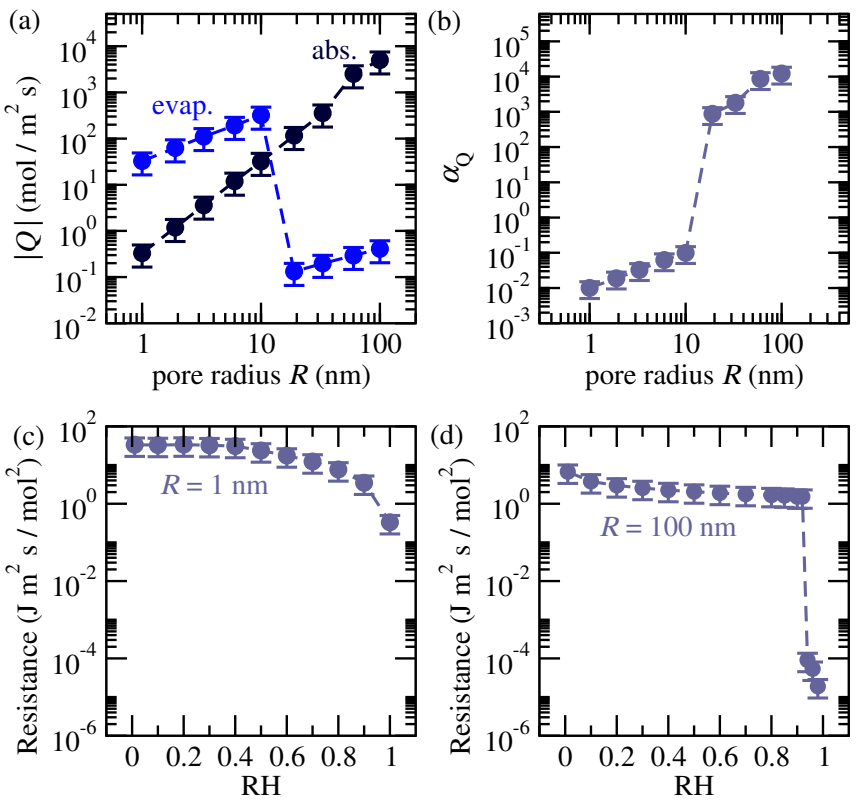

FIG. 7. Transport of water through a micrometric medium. (A) Absolute value of the flux $Q$ during adsorption $(\mathrm{RH}=1)$ and evaporation $(\mathrm{RH}=0.01)$, as a function of the average pore radius $R$. (B) Water flow asymmetry $\alpha_{Q}=|Q(\mathrm{RH}=1) / Q(\mathrm{RH}=0.01)|$ as a function of the average pore radius $R$. (C) Resistance to water transport of the porous medium as a function of the external relative humidity for a small average pore radius $R=1 \mathrm{~nm}$. (D) Resistance for a large average pore radius $R=100 \mathrm{~nm}$.

the density impacts the permeability (Eqs. (6) and (7)), there is about a six-order of magnitude difference between the permeability for evaporation and absorption (Fig.6 C). As a consequence, the membrane shows an absorption flow rate orders of magnitude larger than the evaporation flow rate, respectively $Q_{\text {abs }}=5 \cdot 10^{3} \mathrm{~mol} / \mathrm{s} / \mathrm{m}^{2}$ and $Q_{\text {eva }}=5 \cdot 10^{-1} \mathrm{~mol} / \mathrm{s} / \mathrm{m}^{2}$, corresponding to an asymmetry coefficient $\alpha_{Q}=10^{4}$. As a comparison, we also analyze the water profile within a matrix of small pores of average radius $R=1 \mathrm{~nm}$ (and standard deviation $\Delta R=0.1 \mathrm{~nm}$ ). In this case, the membrane is filled with liquid water in both absorption and evaporation, except at the outer extremity $(x=25 \mu \mathrm{m})$ in the evaporation case (Fig.6B). Accordingly, the permeability is similar in absorption and evaporation, except at the extremity $(x=25 \mu \mathrm{m})($ Fig. $6 \mathrm{D})$, and $Q_{\mathrm{abs}}=3 \cdot 10^{-1} \mathrm{~mol} / \mathrm{s} / \mathrm{m}^{2}$ and $Q_{\text {eva }}=3 \cdot 10^{1} \mathrm{~mol} / \mathrm{s} / \mathrm{m}^{2}$, corresponding to an asymmetry coefficient $\alpha_{Q}=10^{-2}$.

The absorption flux for a liquid scales with the average pore radius $R$ as $\left|Q_{\text {abs }}\right| \propto R^{2}$ while the flux of evaporation scales as $\left|Q_{\text {eval }}\right| \propto R$, except near a critical pore radius, $R_{\mathrm{crt}} \approx 15 \mathrm{~nm}$, where a three order of magnitude jump in flux is measured (Fig.7 $\mathrm{A}$ ). Therefore, $R_{\text {crt }}$ corresponds to the critical average pore radius above which the whole porous medium is filled with water vapor during evaporation regardless of the external chemical potential. For an average pore radius smaller than $R_{\text {crt }}$, the pores located near the osmotic reservoir are filled with liquid water because of capillary condensation. As a consequence of flux conservation within the medium, at equilibrium, most of the porous medium is filled with liquid water during evaporation, regardless of the external chemical potential.

Note that we may have expected the scaling to be $\left|Q_{\text {abs }}\right| \propto R^{4}$ and $\left|Q_{\text {eva }}\right| \propto R^{2}$ since the flux is proportional to the permeability (Eqs. (6) and (7) respectively) multiplied by the pore total surface area $N_{p} \pi R^{2}$, with $N_{p}$ the total number of pores. However the porous medium is of imposed volume $V_{\mathrm{m}}$ and imposed porosity $\epsilon_{p}=0.5=N_{p} \pi R^{2} L_{m} / V_{\mathrm{m}}$. Therefore, the number of pores varies with the average pore radius as $N_{p} \sim R^{-2}$, which affects the scaling of the flux with $R$. For an average pore value $R<R_{\mathrm{crt}}$, the pores are filled with liquid water during evaporation and for $R>R_{\text {crt }}$, the pores are filled with water vapor. As a consequence of the jump of $Q_{\text {eva }}$ near $R=R_{\text {crt }}$, the water flux asymmetry, $\alpha_{Q}=\left|Q_{\text {abs }} / Q_{\text {eva }}\right|$ increases non-linearly with $R$ (Fig.77B). Results show that for a small average pore radius $(R \leq 10 \mathrm{~nm}), \alpha_{Q} \leq 0.1$, and the system losses more water by evaporation than it absorbs over the same period of time. For a large average pore radius $(R>10 \mathrm{~nm})$, the flux asymmetry is $\alpha_{Q} \geq 10^{3}$ (Fig.7BB). Finally, we report the resistance of the porous medium for various external relative humidities $\mathrm{RH}$, for a small average pore radius (Fig. 7 C) and a large average pore radius (Fig.7D). For small pores, the resistances varies with $\mathrm{RH}$ only by about two orders of magnitude, but varies by about 6 orders of magnitude for large pores.

\section{DISCUSSION}

Our results show that a porous medium with pores of average radius $R$ larger than a critical radius $R_{\text {crt }}$ constitutes an efficient water valve when separating an osmotic reservoir from the atmosphere, allowing for a fast absorption of liquid water while limiting its evaporation when exposed to dry air. The main reason for the large transport asymmetry is the difference in fluid content within the porous medium during evaporation and absorption. During evaporation, the medium is filled with low density vapor, while during absorption, it is filled with liquid of comparatively high density. Since the hydraulic resistance of the medium decreases as the fluid density increases, the medium offers little resistance to liquid water but a large resistance to water vapor. For a large pore radius $(R=100 \mathrm{~nm})$, the hydraulic resistance to the transport of water vapor is about five orders of magnitude higher than the resistance to the flow of liquid. Our results also show that a porous medium with small pores $R<R_{\text {crt }}$ forms an inefficient water valve because the pores are mostly filled with liquid water even during evaporation, as a consequence of capillary condensation. 
We also tested a LJ fluid instead of water, and our results show that a porous medium always forms an inefficient valve for a LJ fluid. A crucial difference here between a LJ fluid and water is the ratio between the density of the liquid phase and the density of the vapor phase, typically $\sim 10$ for a LJ fluid, and more than $10^{4}$ for water. As a consequence, in the case of a valve made of an osmotic reservoir of LJ fluid with a reasonable chemical potential, i.e. similar to pure liquid, the porous medium is expected to be mostly filled with high density vapor or even liquid during evaporation. Therefore, the resistance the medium opposes to the evaporation of the LJ fluid is similar to the resistance opposed to liquid absorption. This observation indicates that the valve inspired by the design of Tillandsia's trichome would not be efficient if applied to fluids such as methane, whose properties are close to LJ fluids [50. Finally, our results show that the presence of an adsorbed phase has no impact on the equilibrium transport properties of a medium. However, adsorption modifies the structure of hygrophilic material such as cellulose, with consequences on the transport properties of the medium, see below.

The flow asymmetry coefficient as calculated from simulations includes the contribution from the porous medium only. A realistic valve, however, contains an additional semi-permeable membrane in series with the porous medium (Fig.11). This semi-permeable membrane, which prevents the solutes from spreading through the porous medium, increases the total hydraulic resistance during both absorption and evaporation, which modifies the asymmetry coefficient $\alpha_{Q}$. In order to compare our results with Tillandsia plants, we evaluate the water transport in a system with the same geometry and dimensions as the Tillandsia trichome. The dimensions were extracted from previously published micrographs (see Ref. 9). Using a typical value of resistance for the cell's membrane 51, and that the effective surface area of the foot cell is $150 \mathrm{um}^{2}$, we calculate the resistance of the membrane at the trichome foot $\mathcal{R}_{\mathrm{m}}=3.8 \cdot 10^{-10} \mathrm{~J} \mathrm{~s} / \mathrm{mol}^{2}$. Then, we apply the results obtained for a medium with average pore of radius $R=100 \mathrm{~nm}$ to calculate the resistance of a porous medium with the dimensions of the Tillandia trichome shield. Given that the shield is roughly $21 \mu \mathrm{m}$ thick, with an effective surface area of $650 \mathrm{\mu m}^{2}$, we calculate the resistance of the trichome shield, $\mathcal{R}_{\mathrm{c}}=2.8 \cdot 10^{-12} \mathrm{~J} \mathrm{~s} / \mathrm{mol}^{2}$ in absorption, and $\mathcal{R}_{\mathrm{c}}=1.5 \cdot 10^{-5} \mathrm{~J} \mathrm{~s} / \mathrm{mol}^{2}$ in evaporation $(\mathrm{RH}=0.01)$. From these values, one can deduce a corrected value for the asymmetry coefficient, $\alpha_{Q}=75$, which is of the same order of magnitude as the one measured on Tillandsia plants, $\alpha_{Q}=110$ (Eq. (4)). Note, however, that the present transport model is not aimed at reproducing quantitatively the flow through the shield of the Tillandsia trichome, which would require the use of a realistic pore size distribution. Moreover, the value of $\alpha_{Q}$ as measured on Tillandsia plants has been obtained from whole leaf measurements, and therefore hide potential contributions from the leaf's boundary layer and stomatal transpiration [52].

Our model qualitatively reproduces the effects of relative humidity variations on the resistance of Tillandsia trichomes (Fig.7D and Fig.11B). In both cases, the resistance is quasi constant below a certain humidity threshold $\left(\mathrm{RH}^{*} \approx 0.8\right.$ for the plant and $\mathrm{RH}^{*} \approx 0.9$ for the model) but drops drastically for $\mathrm{RH}>\mathrm{RH}^{*}$. The drop near $\mathrm{RH}^{*}$ is smoother in the case of the plant, which is probably due to the much broader pore size distribution 53 as compared to the one used in the model. Note also that due to the semi-permeable membranes which is not present in the model, the drop in resistance near $\mathrm{RH}^{*}$ as calculated from the model is about 6 orders of magnitude, but only about 4 orders of magnitude for the plant.

In most of this study, interactions between the structure of the pore and the fluid have been neglected. But cellulose-based materials swell when their internal water content is increased due to the interaction between water and the hydroxyl groups of the cellulose [54. Reported values of swelling for cotton corrected for lumen area are about 31-33\% [55. Molecular dynamics simulations of cellulose-based materials show a linear increase of the porosity with the moisture content [56]. Using these values for cellulose materials, we can estimate that a medium with cylindrical pores of radius $R=100 \mathrm{~nm}$ when fully swollen, would have pores with radius of $R \approx 82 \mathrm{~nm}$ after shrinking. The pore size reduction would induce a reduction of the evaporating flux by about $30 \%$, and accordingly lead to an increase of the asymmetry coefficient $\alpha_{Q}$. In addition, the swelling of the cellulose is usually associated with a decrease in tortuosity following pore expansion and merging, leading to an even larger asymmetry coefficient.

Here we considered only cylindrical pores, but cellulosebased materials are fibrous. Fibrous materials have a permeability to fluid with similar scaling in density and pore dimension as cylinder pores, with however different prefactors [57, 58. Hence, the flux predicted by our model on cylindrical pores may differ from what would be obtained using a more realistic fibrous medium. However, the predicted ratio between absorption and evaporation flux should remain similar.

In this study, the flux of latent heat associated with the phase change during evaporation has been neglected. This flux reduces the temperature of the osmotic reservoir from which evaporation occurs, and in turn reduces the rate of evaporation. From a balance between a net flux of density of radiation $\mathcal{R}$, a sensible heat flux density $\mathcal{C}$ and a flux of latent heat $\mathcal{L}=h_{\text {vap }} Q_{\text {eva }}$ with $h_{\text {vap }}$ the enthalpy of vaporization, we estimate that thermal effects reduce the flow $Q_{\text {eva }}$ by about $10 \%$ [59, 60], hence increasing the asymmetry coefficient $\alpha_{Q}$. 


\section{CONCLUSION}

transport asymmetry.

\section{ACKNOWLEDGMENTS}

We use a multi-scale fluid transport model to mimic the function of Tillandsia trichomes. We show that a porous medium with pore dimension larger than a threshold can constitute an efficient passive water valve when separating an osmotic reservoir from the atmosphere; allowing for the fast absorption of liquid water while also limiting the evaporation of water vapor when the atmosphere is dry. The difference in transport properties between the vapor and liquid phases has been identified as the basis for the
SG acknowledges support from Fondecyt's postdoctoral fellowships \#3170476. This research was supported in part by grant \#1130129 from Fondecyt (Chile) and the US Army Research Development and Engineering Command (RDECOM), the US Office of Naval Research (ONR), and the US Air Force Office of Scientific Research (AFOSR) under grant number W911NF-16-1-0434 (\$194,329 USD). The authors thank M. Stojanova for comments on the manuscripts.
[1] C. J. Vorosmarty, "Global Water Resources: Vulnerability from Climate Change and Population Growth," Science 289, 284-288 (2000)

[2] Syed Akhlaq Ahmad and Sohail Rasool Lone, "Hybrid Process ( Pervaporation-Distillation ): A Review," International Journal of Scientific \& Engineering Research 3, 1-5 (2012).

[3] K.S. Spiegler and O. Kedem, "Thermodynamics of hyperfiltration (reverse osmosis): criteria for efficient membranes," Desalination 1, 311-326 (1966)

[4] C. Fritzmann, J. Löwenberg, T. Wintgens, and T. Melin, "State-of-the-art of reverse osmosis desalination," Desalination 216, 1-76 (2007).

[5] J.L. Monteith, "Dew," Quarterly Journal of the Royal Meteorological Society , 322-341 (1957)

[6] Christopher P. McKay, E. I. Friedmann, B. Gomez-Silva, L. Caceres-Villanueva, D. T. Andersen, and R. Landheim, "Temperature and Moisture Conditions for Life in the Extreme Arid Region of the Atacama Desert: Four Years of Observations Including the El Nino of 1997-1998," Astrobiology 3, 393-406 (2003)

[7] P. Cereceda, H. Larrain, P. Osses, M. Farías, and I. Egaña, "The spatial and temporal variability of fog and its relation to fog oases in the Atacama Desert, Chile," Atmospheric Research 87, 312-323 (2008)

[8] Marc Muselli, Daniel Beysens, Jacques Marcillat, Irina Milimouk, Torbjorn Nilsson, and Alain Louche, "Dew water collector for potable water in Ajaccio (Corsica Island, France)," Atmospheric Research 64, 297-312 (2002).

[9] Pascal S Raux, Simon Gravelle, and Jacques Dumais, "Design of a Unidirectional Water Valve in Tillandsia," (Accepted for publication in Nature Communication) $\mathbf{0}$ (2019).

[10] Philip W Rundel, Beatriz Palma, Michael 0 Dillon, M Rasoul Sharifi, Erik T Nilsen, Kansri Boonpragob, and Julio R Gutierrez, "Tillandsia landbeckii in the coastal Atacama Desert of northern Chile," Revista Chilena de Historia Natural 70, 341-349 (1997).

[11] Carl Mez, "Die Wasser-Okonomie der extrem atmospherischen Tillandsien," Physiologische BromeliaceenStudien (1904).

[12] D. H. Benzing, "Bromeliaceae: Profile of an Adaptive Radiation." Edinburgh Journal of Botany 59, 325-334 (2000).
[13] Jan C T Eijkel and Albert van den Berg, "Water in micro- and nanofluidics systems described using the water potential." Lab on a chip 5, 1202-1209 (2005).

[14] G. Job and F. Herrmann, "Chemical potential - A quantity in search of recognition," European Journal of Physics 27, 353-371 (2006)

[15] Park S Nobel, Physicochemical and environmental plant physiology (Academic press, 1999).

[16] Alain Cloutier and Yves Fortin, "A model of moisture movement in wood based on water potential and the determination of the effective water conductivity," Wood Science and Technology 27, 95-114 (1993)

[17] Carl Tremblay, Alain Cloutier, and Yves Fortin, "Determination of the effective water conductivity of red pine sapwood," Wood Science and Technology 34, 109-124 (2000)

[18] S. L. Berry and M. L. Roderick, "Plant-water relations and the fibre saturation point," New Phytologist 168, 25-37 (2005)

[19] Jean-Mathieu Vanson, François-Xavier Coudert, Benjamin Rotenberg, Maximilien Levesque, Caroline Tardivat, Michaela Klotz, and Anne Boutin, "Unexpected coupling between flow and adsorption in porous media," Soft matter 11, 6125-6133 (2015).

[20] AlfredJ Stamm, "Movement of $1^{\sim}$ luids in Wood - Part II: Diffusion," Wood Science and Technology 1, 205-230 (1967)

[21] S Plimpton, "Fast Parallel Algorithms For Short-range Molecular-dynamics," J. Comp. Phys. 117, 1-19 (1995)

[22] Suresh K. Bhatia and David Nicholson, "Hydrodynamic Origin of Diffusion in Nanopores," Physical Review Letters 90, 4 (2003)

[23] J. A. Weaver and A. B. Metzner, "The surface transport of adsorbed molecules," AIChE Journal 12, 655-661 (1966)

[24] D J Adams, "Chemical potential of hard-sphere fluids by Monte Carlo methods," Molecular Physics 28, 1241-1252 (1974).

[25] Daan Frenkel and Berend Smit, Understanding Molecular Simulation (Elsevier, 2001).

[26] Suresh K. Bhatia, Owen Jepps, and David Nicholson, "Tractable molecular theory of transport of Lennard-Jones fluids in nanopores," Journal of Chemical Physics 120, 4472-4485 (2004) 
[27] Karl P. Travis and Keith E. Gubbins, "Combined diffusive and viscous transport of methane in a carbon slit pore," Molecular Simulation 25, 209-227 (2000)

[28] Karsten Meier, Arno Laesecke, Stephan Kabelac, Karsten Meier, and Stephan Kabelac, "Transport coefficients of the Lennard-Jones model fluid . I . Viscosity Transport coefficients of the Lennard-Jones model fluid . I . Viscosity," The Journal of Chemical Physics 3671 (2004), 10.1063/1.1770695.

[29] Martin Knudsen, "The law of molecular flow and viscosity of gases moving through tubes," Ann. Phys. 28, 75 (1909).

[30] von M. v. Smoluchowski, "Zur kinetischen Theorie der Transpiration und Diffusion verdunnter Gase," Annalen der Physik 338 (1910).

[31] E A Mason, A P Malinauskas, and R B Evans, "Flow and Diffusion of Gases in Porous Media," Journal of chemical physics 46 (1967), 10.1063/1.1841191

[32] Fabrizio Capuani, Ignacio Pagonabarraga, and Daan Frenkel, "Discrete solution of the electrokinetic equations," Journal of Chemical Physics 121, 973-986 (2004) arXiv:0404289v1 [arXiv:cond-mat]

[33] Alexandru Boţan, Franz Josef Ulm, Roland J.M. Pellenq, and Benoit Coasne, "Bottom-up model of adsorption and transport in multiscale porous media," Physical Review E - Statistical, Nonlinear, and Soft Matter Physics 91, $1-10$ (2015).

[34] Shiyi Chen and Gary D. Doolen, "Lattice Boltzmann Method," Annu. Rev. Fluid Mech. 30, 329-64 (1998)

[35] Alberto Striolo, "The mechanism of water diffusion in narrow carbon nanotubes," Nano Letters 6, 633-639 (2006).

[36] J. L. Abascal and C. Vega, "A general purpose model for the condensed phases of water: TIP4P/2005." The Journal of chemical physics 123, 234505 (2005)

[37] Karl N. Kirschner, Austin B. Yongye, Sarah M. Tschampel, Jorge González-Outeiriño, Charlisa R. Daniels, B. Lachele Foley, and Robert J. Woods, "GLYCAM06: A Generalizable Biomolecular Force Field. Carbohydrates," Journal of computational chemistry 29, 622-655 (2008) arXiv:NIHMS150003.

[38] Thiago C F Gomes and Munir S. Skaf, "Cellulose-builder: A toolkit for building crystalline structures of cellulose," Journal of Computational Chemistry 33, 1338-1346 (2012).

[39] Yoshiharu Nishiyama, Junji Sugiyama, Henri Chanzy, and Paul Langan, "Crystal Structure and Hydrogen Bonding System in Cellulose I $r$ from Synchrotron X-ray and Neutron Fiber Diffraction," J. Am. Chem. Soc. 125, 14300-14306 (2003).

[40] Karol Kulasinski, Robert Guyer, Dominique Derome, and Jan Carmeliet, "Poroelastic model for adsorption-induced deformation of biopolymers obtained from molecular simulations," Physical Review E - Statistical, Nonlinear, and Soft Matter Physics 92, 1-10 (2015)

[41] Jean-Paul Ryckaert, Giovanni Ciccotti, and Herman J.C Berendsen, "Numerical integration of the cartesian equations of motion of a system with constraints: molecular dynamics of n-alkanes," Journal of Computational Physics 23, 327-341 (1977)

[42] Benoit Coasne, Anne Galarneau, Francesco Di Renzo, and Roland J.M. Pellenq, "Molecular simulation of adsorption and intrusion in nanopores," Adsorption 14, 215-221 (2008).
[43] C. Vega and E. De Miguel, "Surface tension of the most popular models of water by using the test-area simulation method," Journal of Chemical Physics 126 (2007), $10.1063 / 1.2715577$

[44] Minoru Miyahara, Hideki Kanda, Tomohisa Yoshioka, and Morio Okazaki, "Modeling capillary condensation in cylindrical nanopores: a molecular dynamics study," Langmuir 16, 4293-4299 (2000)

[45] Richard C. Tolman, "The effect of droplet size on surface tension," The Journal of Chemical Physics 17, 333-337 (1949)

[46] Mingyang Chen, Benoit Coasne, Robert Guyer, Dominique Derome, and Jan Carmeliet, "Role of hydrogen bonding in hysteresis observed in sorption-induced swelling of soft nanoporous polymers," Nature Communications 9 (2018), 10.1038/s41467-018-05897-9

[47] Miguel Angel González and Joś L F Abascal, "The shear viscosity of rigid water models," Journal of Chemical Physics 132, 096101 (2010).

[48] Pierfranco Demontis and Giuseppe B. Suffritti, "A comment on the flexibility of framework in molecular dynamics simulations of zeolites," Microporous and Mesoporous Materials 125, 160-168 (2009).

[49] A. Ghoufi and G. Maurin, "Hybrid monte carlo simulations combined with a phase mixture model to predict the structural transitions of a porous Metal-organic framework material upon adsorption of guest molecules," Journal of Physical Chemistry C 114, 6496-6502 (2010)

[50] H Docherty, a Galindo, C Vega, and E Sanz, "A potential model for methane in water describing correctly the solubility of the gas and the properties of the methane hydrate." The Journal of chemical physics 125, 074510 (2006)

[51] Christophe Maurel, "Aquaporins and water permeability of plant membranes," Annual Review of Plant Physiology and Plant Molecular Biology 48, 399-429 (1997).

[52] O. L. Lange and E. Medina, "Stomata of the CAM plant Tillandsia recurvata respond directly to humidity," Oecologia 40, 357-363 (1979)

[53] Michael Plotze and Peter Niemz, "Porosity and pore size distribution of different wood types as determined by mercury intrusion porosimetry," European Journal of Wood and Wood Products 69, 649-657 (2011)

[54] Chen, Zhang, Shomali, Coasne, Carmeliet, and Derome, "Wood-Moisture Relationships Studied with Molecular Simulations: Methodological Guidelines," Forests 10, 628 (2019)

[55] G. I. Mantanis, R. A. Young, and R. M. Rowell, "Swelling of compressed cellulose fiber webs in organic liquids," Cellulose 2, 1-22 (1995).

[56] Karol Kulasinski, Robert Guyer, Dominique Derome, and Jan Carmeliet, "Water Diffusion in Amorphous Hydrophilic Systems: A Stop and Go Process," Langmuir 31, 10843-10849 (2015)

[57] Graham W. Jackson and David F. James, "The Permeability of Fibrous Porous Media," The Canadian Journal of Chemical Engineering 64, 365-374 (1986).

[58] Dahua Shou, Gas and Vapor Transport through Nano- and Micro- Fi Ph.D. thesis, The Hong-Kong Polytechnic University (2012).

[59] David M. Gates, "Transpiration and leaf temperature," Annual Review of Plant Physiology 19, 211-238 (1968).

[60] J.L. Monteith, "Evaporation and surface temperature," Quarterly Journal of the Royal Meteorological Society 107, 1-27 (1981). 